\title{
Determination of kinematic distances of pre-main-sequence stars in star-forming regions
}

\author{
Phillip A. B. Galli, ${ }^{1}$ Ramachrisna Teixeira, ${ }^{1}$ Christine Ducourant ${ }^{2}$ \\ and Claude Bertout ${ }^{3}$ \\ ${ }^{1}$ Instituto de Astronomia, Geofísica e Ciências Atmosféricas, Universidade de São Paulo, \\ Rua do Matão 1226, Cidade Universitária, São Paulo, Brazil \\ email: galli@astro.iag.usp.br \\ ${ }^{2}$ Laboratoire d'Astrophysique de Bordeaux, Université de Bordeaux I, 2 Rue de l'Observatoire, \\ 33270 Floirac, France \\ ${ }^{3}$ Institut d'Astrophysique de Paris, 98bis Bd. Arago, 75014, Paris, France
}

\begin{abstract}
Many studies of star-forming regions have been carried out since the discovery of compact HII regions in the late 1960s. The kinematic properties of young stars in the nearest regions with ongoing and recent star formation provide essential tests of their formation mechanisms. The detection of coeval moving groups allows determination of individual distances through the convergent-point method. As a result, the main physical properties of these stars and their early evolutionary stages can be determined if we know how distant they are.
\end{abstract}

Keywords. stars: distances, stars: kinematics, stars: evolution, stars: formation

\section{Introduction}

A precise determination of distances is required to accurately determine the main physical parameters of stars (mass, age, luminosity and temperature) and to trace the evolution of young stars. Kinematic studies of young stars based on distances, proper motions and radial-velocity information are fundamental to investigate their formation mechanisms. Young stellar objects, including pre-main-sequence (PMS) stars, are supposed to be very close to their birth places and have velocities still very similar to the initial values, so that one can get clear constraints on the birth mechanisms. Many PMS stars were not observed by the Hipparcos satellite because of their faint magnitudes nor do they have any trigonometric parallaxes measured from the ground due to their distances. In this context, knowledge of the proper motions of young stars is essential because they allow detection of structures such as moving groups and associations to which these stars belong. The members of a moving group, which share the same space motion, allow us to obtain the convergent point from which we can determine the kinematic distance if the radial velocity is known.

\section{The sample of pre-main-sequence stars}

We have a sample of 1250 PMS stars and 104 candidates, spread over major starforming regions (SFRs), as shown in Figure 1, taken from the proper-motion catalogue of PMS stars Ducourant et al. (2005). An exhaustive search in the literature for PMS stars in the proper-motion catalogue with known radial velocities has been conducted to develop our strategy. So far, we have found radial-velocity values for 410 stars, corresponding 
to $30 \%$ of our sample. Likewise, we searched for PMS stars with known parallaxes to compare with our future results. We found parallax values for 58 stars, corresponding to $4 \%$ of our sample. The main source for parallaxes is the Hipparcos catalogue.

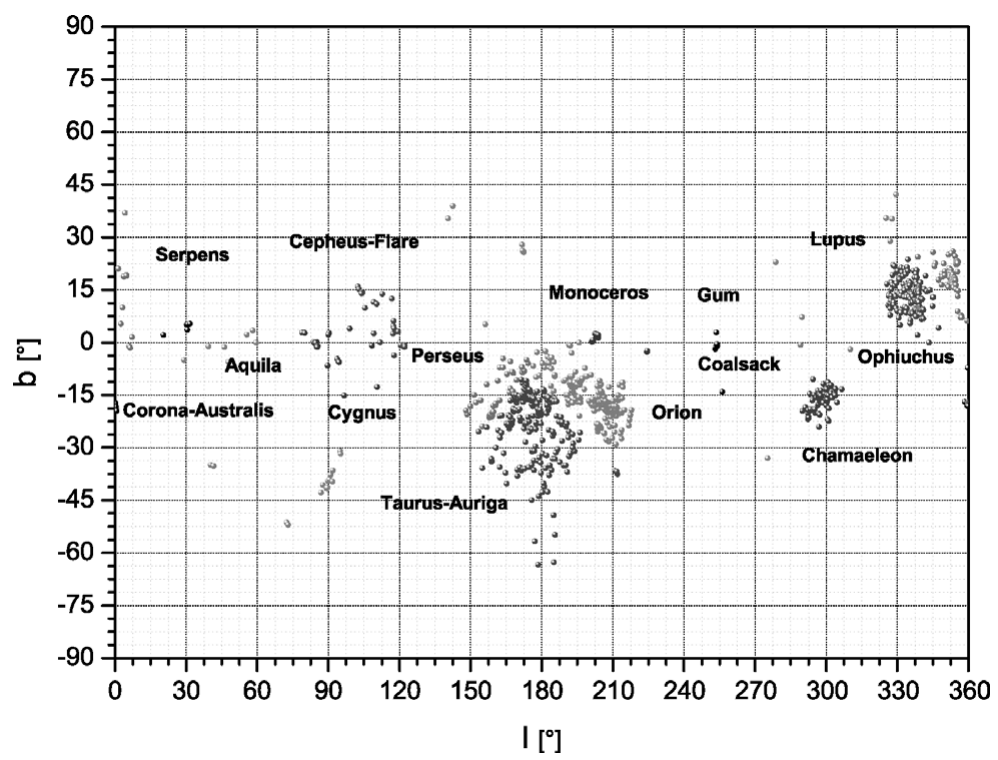

Figure 1. Sky distribution of the SFRs in the proper-motion catalogue of PMS stars.

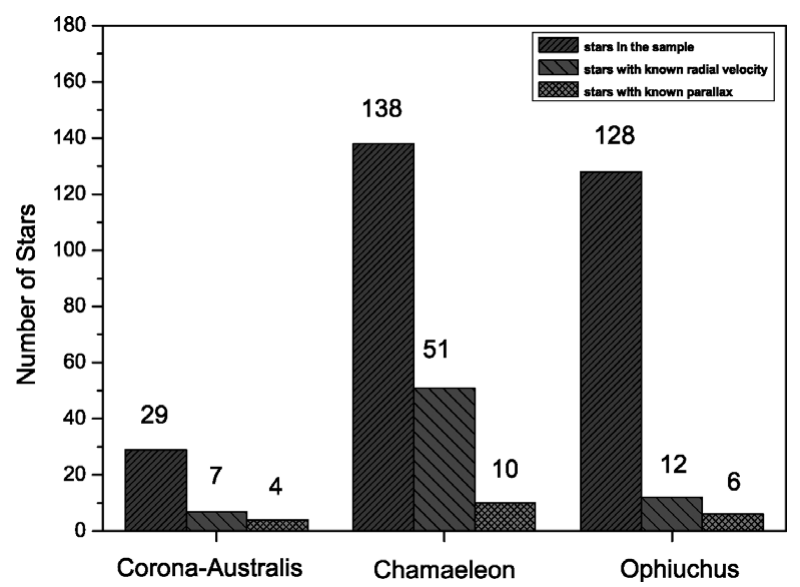

Figure 2. Brief census of the Corona Australis, Chamaeleon and Ophiuchus star-forming regions. We present the total number of stars in each star-forming region compared with the number of stars that have radial-velocity and parallax information.

This work will focus on a kinematic study of three SFRs and the same strategy developed here may be applied to the other regions in the future. Our priority list includes the following SFRs: Corona Australis, Chamaeleon and Ophiuchus. A brief census of each SFR considered is presented in Figure 2. 


\section{Convergent-point analysis}

Although average distances for a moving group provide valuable information, what we really need for evolutionary models are the distances to individual stars in the group. The procedure that we use here to derive individual parallaxes is based on a knowledge of radial velocities and on the convergent-point method which makes full use of directly observed astrometric data, i.e., positions and proper motions. We developed our first convergent-point analysis based on Mamajek (2005). In this implementation, we approximately follow the convergent-point grid technique of Jones (1971), using the definition of $t^{2}$ and velocity dispersion of de Bruijne (1999a). The convergent-point code has been applied to the TW Hydrae association for a check of our results. A comparison among convergent-point analyses from different authors is shown in Figure 3. We use the TW Hydrae association only as a check of our data processing. The whole strategy developed here will be applied to the PMS stars in the Corona Australis, Chamaeleon and Ophiuchus star-forming regions.

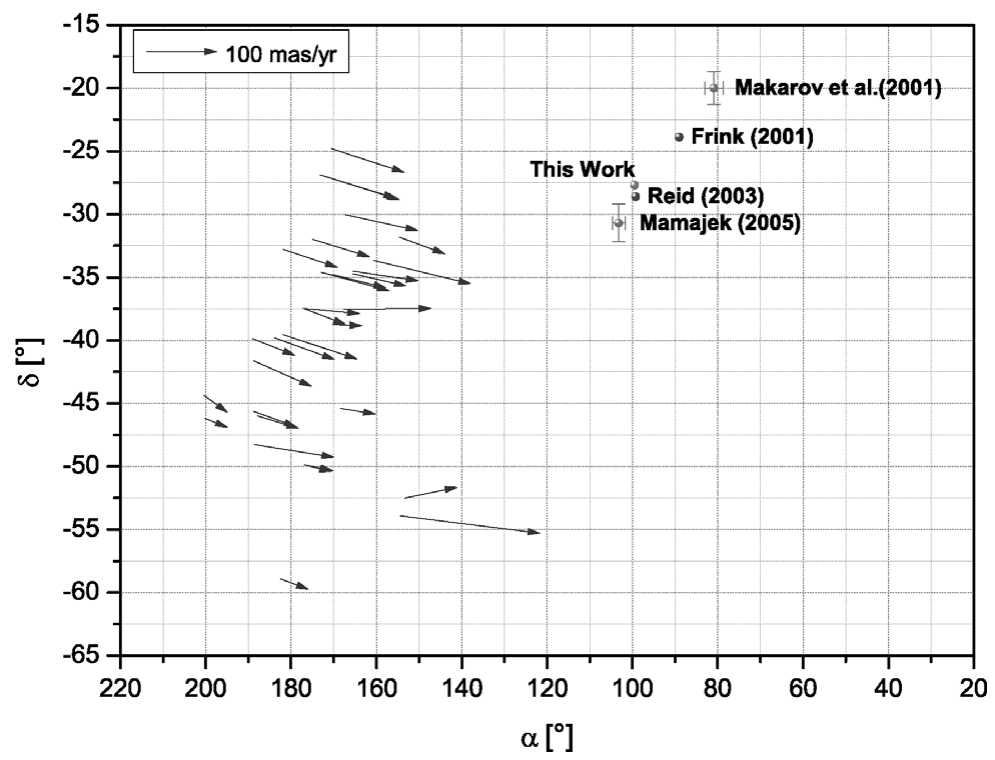

Figure 3. Comparison of convergent-point analysis for the TW Hydrae association.

The current stage of this work concerns the evaluation of the convergent point's uncertainty, of which the determination is not clear in the literature. As soon as we have finished the implementation of our own convergent-point strategy, we will be able to evaluate the kinematic distance to each group member considering that its radial velocity is known:

$$
\Pi_{\text {ind }}=\frac{4.74 \mu}{V_{\text {rad }} \tan \lambda}
$$

The next step is dedicated to application of the distances derived here and analysis of the kinematic properties of each SFR. This work is the initial part of a more complete project which represents an effort to better constrain the early stages of stellar evolution as regards the formation mechanism, disk lifetime, timescale of planet formation and kinematics of young stellar objects. 


\section{References}

Bertout, C. \& Genova, F. 2006, A\& A, 460, 499

Bertout, C., Siess, L., \& Cabrit, S. 2007, A\&A (Letters), 473, L21

de Bruijne, J. H. J. 1999, MNRAS, 306, 381

Ducourant, C., Teixeira, R., Périé, J. P., Lecampion, J. F., Guibert, J., \& Sartori, M. J. 2005, $A \mathscr{S} A, 438,769$

Frink, S. 2001, in: R. Jayawardhana \& T. Greene (eds.), Young Stars Near Earth: Progress and Prospects, in ASP Conf. Ser., 244, p. 16

Jones, D. H. P. 1971, MNRAS, 152, 231

Makarov, V. V. \& Fabricius, C. 2001, A\& $A, 368,866$

Mamajek, E. E. 2005, ApJ, 634, 1385

Reid, N. 2003, MNRAS, 342, 837

Teixeira, R., Ducourant, C., Sartori, M. J., Camargo, J. I. B., Périé, J. P., Lépine, J. R. D., \& Benevides-Soares, P. 2000, A\&\&A, 361, 1143 